\title{
POLÍTICAS PÚBLICAS DE SAÚDE NA ODONTOLOGIA: RESGATE HISTÓRICO E SUA RELAÇÃO COM A DISFUNÇÃO TEMPOROMANDIBULAR
}

\author{
Laio da Costa DUTRA ${ }^{1}$ \\ Eduardo José Guerra SEABRA ${ }^{2}$ \\ Isabela Pinheiro Cavalcanti LIMA ${ }^{3}$ \\ Alexandre Policarpo da SILVA ${ }^{4}$ \\ Yuri Victor de Medeiros MARTINS ${ }^{5}$
}

\begin{abstract}
${ }^{1}$ Cirurgião-dentista. Mestrando do Programa de Pós-Graduação em Saúde e Sociedade da Universidade do Estado do Rio Grande do Norte - PPGSS/UERN. Professor Substituto do Curso de Odontologia da Universidade do Estado do Rio Grande do Norte - UERN. Email: laiodutra@gmail.com
\end{abstract}

${ }^{2}$ Cirurgião-dentista. Doutor em Ciências da Saúde pela Universidade Federal do Rio Grande do Norte - UFRN. Professor Adjunto IV do Curso de Odontologia da Universidade do Estado do Rio Grande do Norte. Email: ejgseabra@yahoo.com.br.

${ }^{3}$ Cirurgiã-dentista Doutora em Ciência e Engenharia de Materiais pela Universidade Federal do Rio Grande do Norte UFRN. Professora do Curso de Odontologia da Universidade do Estado do Rio Grande do Norte - UERN. Email: belapcl@yahoo.com.br.

${ }^{4}$ Cirurgião-dentista. Mestrando do Programa de Pós-Graduação em Saúde e Sociedade da Universidade do Estado do Rio Grande do Norte - PPGSS/UERN. Professor Substituto do Curso de Odontologia da Universidade do Estado do Rio Grande do Norte - UERN. Email: policarpogande@ hotmail.com

${ }^{5}$ Cirurgião-dentista. Mestrando do Programa de Pós-Graduação em Saúde e Sociedade da Universidade do Estado do Rio Grande do Norte - PPGSS/UERN. Email: yurivictormm@gmail.com

Recebido em: 30/05/2014 - Aprovado em: 18/09/2014 - Disponibilizado em: 15/12/2014

RESUMO: O objetivo deste estudo é através uma revisão de literatura,analisar e abordar os aspectos sociais, econômicos, políticos e culturais durante a história da odontologia e sua relação com as políticas e práticas de saúde bucal no Brasil, assim como apresentar uma das consequências da prática desenvolvida até os dias atuais. Foram utilizadas as bases de dados eletrônicos Bireme, Pubmed, como também livros, dissertações, teses e referências legislativas. Foram criadas várias políticas de saúde bucal ao longo dos anos para proporcionar um melhor atendimento odontológico à população. Contudo, dentro das consequências destas ações destacam-se as várias perdas dentárias ao longo do tempo. Como resultado, pode-se ocorrer o surgimento de situações danosas à população, dentre elas quadros de disfunção temporomandibular.Esta associação necessita de estudos, pois é pouco relatada nas bases de dados eletrônicos e em outros meios de publicação literária, assim há uma carência de estudos sobre este assunto.

Palavras-chave: História da Odontologia. Perda de Dente. Síndrome da Disfunção da Articulação Temporomandibular.Desordens temporomandibulares.Boca Edentada.

\section{Public health policies in dentistry: historical review and its relation to temporomandibular dysfunction}

\begin{abstract}
The aim of this study is through a literature review, analyze and approach the social, economic, political and cultural aspects in the history of dentistry and its relationship with the policies and practices of oral health in Brazil, as well as presenting one of the consequences the practice developed to the present day. The electronic databases Bireme, Pubmed, as well as books, dissertations, theses and legal references were used . Several dental health policies were created over the years to provide better dental care to the population. However, these actions caused as a result of various tooth loss over time. As a consequent, can occur the emergence of harmful situations the population, including cases of temporomandibular dysfunction .This association needs studies, it is rarely reported in the electronic databases and other means of literary publishing, developing a dearth of studies on the subject.

Keywords:History of Dentistry. Tooth Loss. Temporomandibular Joint Dysfunction Syndrome.Edentulous mouth.
\end{abstract}




\section{INTRODUÇÃO}

Percebe-se que a assistência odontológica no Brasil era inexistente para a população no início do processo de colonização. A atenção odontológica somente ocorreu com a chegada dos colonizadores, pois além destes, vieram ao país pessoas que prestavam atendimento odontológico. Este era somente realizado para os colonizadores, deixando a população sem nenhuma forma de atendimento. Posteriormente, a assistência odontológica iniciou-se a partir dos barbeiros, que eram pessoas que aprendiam o ofício da arte dentária, como era conhecida na época, e realizavam atendimentos a população. A atividade desenvolvida pelo barbeiro era arbitrária e de fácil compreensão, sendo necessário pouco conhecimento teórico, tornando-o adequado aos escravos, pois era uma forma fácil de inserção no mercado de trabalho (CARVALHO, 2003).

No início do século XX, devido à economia ser agroexportadora, o país direcionou as políticas de saúde para os espaços de comercialização das mercadorias produzidas, sem dar ênfase à saúde do trabalhador. Quando este modelo econômico entra em crise,o foco das políticas passa a ser o de cuidar dos trabalhadores.Assim, cria-se uma política compensatória aos trabalhadores, por meio de um sistema previdenciário (ao qual estava vinculado as ações de saúde), conhecida como Caixas de Aposentadorias e
Pensões (CAPs) e em seguida os Institutos de Aposentadorias e Pesões (IAPs) (RONCALLI, 2003).A assistência odontológica tem inicio neste período, visando principalmente os atendimentos emergenciais(LOURENÇO, 2003), sem dar ênfase à parte de promoção e prevenção em saúde bucal para a população.Esta assistência,no final da década de 1970, começou a lutar para que fossem implementadas políticas de saúde baseadas no principio da universalidade. Além da população em geral, profissionais de saúde e intelectuais da área de saúde coletiva aderiram ao movimento que posteriormente seria conhecido como Movimento pela Reforma Sanitária (RONCALLI, 2003). Este período coincide com o declínio das concepções socialistas ou de estado de bem-estar em todo o mundo, sendo argumentado e defendido a focalização dos programas sociais, e dentro dos princípios defendidos pelo Sistema Único de Saúde (SUS) seria o de abrangência universal (CAMPOS, 2006).O objetivo deste artigo é, através de uma revisão de literatura, abordar os aspectos sociais, econômicos, políticos e culturais durante a história da odontologia, como também a sua relação com as políticas e práticas de saúde bucal no Brasil. O artigo também apresenta uma das consequências da prática desenvolvida desde o período colonial aos dias atuais. Para a construção deste trabalho, foram utilizadas as bases de dados eletrônicos Bireme, Pubmed, 
como também livros, dissetações, teses e texto legislativos.

\section{REVISÃO DE LITERATURA}

\section{A PRÁtica dA ODONTOLOGIA NO PERÍODO COLONIAL}

Após o processo de colonização vieram ao país indivíduos de várias profissões, visando desenvolver serviços e cuidados as pessoas responsáveis por este processo (SILVA; SALES-PERES, 2007), fazendo com que a população que não fazia parte deste seleto grupo não tivesse acesso ao tratamento de suas necessidades de saúde bucal. Desde o início da colonização, a responsabilidade pelo atendimento a maior parte da população era realizada pelas Santas Casas de Misericórdia, e nesta época a odontologia praticava quase que exclusivamente extrações dentárias(LOURENÇO, 2003),contribuindo para um maior número de pessoas sem dentes.Desta forma, as pessoas buscavam na maioria das vezes, devido à dificuldade de atendimento, a resolução das alterações por eles acometidas através de benzeduras, rezas, pólvora e teias de aranha (PEREIRA, 2012).

Em 09 de novembro de 1629 houve, por meio de Carta Régia, os exames aos cirurgiões e barbeiros, e posteriormente criou-se a reforma do regimento, em 12 de dezembro de 1631, que estabeleceu penalidades em forma de multa no valor de dois mil réis às pessoas que "tirassem dentes" sem a licença adquirida por meio dos exames (LOURENÇO, 2003). A odontologia no período colonial não era considerada como uma atividade científica, pois era desenvolvida como um anexo da Medicina, e praticada por barbeiros, cirurgiões ou por práticos sem possuírem formação para exercer esta profissão (PEREIRA, 2012), desenvolvendo-a de forma empírica.

Algumas

nomenclaturas, de acordo com a época e o contexto social inserido, deram um significado as atividades desenvolvidas pelos profissionais em seu trabalho na saúde bucal: barbeiros, curandeiros, pseudodentistas, sangradores, tira-dentes, barbeiroscirurgiões(WARMLING;

CAPONI; BOTAZZO, 2006). O individuo conhecido como tira-dentes possuía uma caixa de horrores (faca de vários tamanhos e formas, alavancas), em que este somente era consultado quando nenhuma outra possibilidade fosse possível(PEREIRA, 2012).

No final do século XVIII, Joaquim José da Silva Xavier (1746-1792) desenvolveu a Odontologia que aprendeu com seu padrinho, Sebastião Ferreira Leitão, em que neste período as exodontias eram realizadas com as chaves de Garangeot, alavancas rudimentares, e o pelicano, realizando as obturações em chumbo sobre o 
tecido com lesão de cárie e polpas dentárias afetadas, com consequências danosas (LOURENÇO, 2003).Mesmo utilizando remédios caseiros e fórmulas secretas (geralmente comercializadas pelos charlatães), a forma mais comum e praticada de solucionar a dor de dentes era através da extração dentária (CARVALHO, 2003).

Na segunda metade do século XIX, a prática de forma legal da arte dentária, assim como era conhecida na época, tanto por dentistas brasileiros como também por estrangeiros, era necessário à aprovação em um exame de habilitação realizado apenas pelas Faculdades de Medicina do Rio de Janeiro e da Bahia. (MOTTet al., 2008). Assim, teve início o processo de controle da atividade, buscando o desenvolvimento da odontologia por meio de pessoas com algum nível de qualificação, alterando a conjuntura anterior, pautada na prática odontológica sem habilitação para exercer a profissão.

A odontologia por representar a idéia de arte dentária como profissão para a população dificultou o seu reconhecimento como atividade e meio para formação científica, pois devido a grande necessidade de profissionais e um número insuficiente de dentistas formados no inicio dos anos de 1900, fizeram com que a quantidade de dentistas sem formação atuando aumentasse (PEREIRA, 2012).Mesmo com a hegemonia do enfoque médico biológico no inicio da saúde pública, a tensão entre estes e os enfoques social e político como também ambiental ainda foram presentes durante $\mathrm{O}$ século XX (BUSS; PELLEGRINI FILHO, 2007).

\section{ODONTOLOGIA NO INÍCIO DO SÉCULO XX AOS ANOS DE 1980 NO BRASIL}

Devido no início do século $\mathrm{XX}$ a economia brasileira era essencialmente agrícola, dependendo economicamente da exportação, em especial de café e açúcar, a preocupação foi direcionada aos espaços onde ocorria a circulação de mercadorias, assim as ações desenvolvidas foram o saneamento dos portos (RONCALLI, 2003). Neste período, somente era de interesse o controle das doenças nos espaços de comercializações da época, portanto a atenção do governo brasileiro foi direcionada para os chamados "espaços de circulação de mercadorias", ou seja, as estradas e os portos, principalmente os do Rio de Janeiro e de Santos, em que ocorria a maior parte do transporte e comercialização dos produtos agrícolas (VARGAS, 2008).Dentre umas das iniciativas do inicio do século XX, destacou-se a realizada pelo então presidente Rodrigues Alves que, em 1902, criou o programa de saneamento do Rio de Janeiro e o combate à febre amarela em São Paulo (RONCALLI, 2003), pois ocorreu preocupação por parte dos governantes da época em função das epidemias que se 
alastravam nestas cidades. Este tipo de modelo de intervenção desenvolvido por Oswaldo Cruz ficou conhecido como campanhista, e foi concebido dentro de uma visão militar. Mesmo sendo realizado de forma arbitrária e com abusos, o modelo campanhista obteve importantes resultados positivos no controle das doenças epidêmicas, atingindo inclusive a erradicação da febre amarela da cidade do Rio de Janeiro, o que fortaleceu este modelo e o tornou hegemônico, intervindo na saúde pública durante os anos seguintes(VARGAS, 2008).

Com a mudança no modelo econômico do país, as exportações diminuíram e se iniciou a industrialização, sendo necessário trazer mão-de-obra especializada, principalmente europeia. Estes sofriam com as doenças contagiosas, levando-os a se organizarem e exigirem melhorias à sua saúde. Com isso se iniciou as ações assistenciais direcionadas aos trabalhadores, vinculadas ao sistema previdenciário.

Posteriormente a revolução de 1930, um novo período na política se estabelece, pois com o crescimento político de Getúlio Vargas e a queda das oligarquias, ocorre uma reforma administrativa e política resultando na nova Constituição de 1934 e a ditadura imposta por Vargas com o Estado Novo em 1937, sendo este período acompanhado de uma mudança no modelo econômico que irá altera $\mathrm{o}$ foco de atuação da assistência (RONCALLI, 2003). A queda das oligarquias gera ao modelo agroexportador impactos negativos, ajudando a modificar, desta forma, as necessidades de saneamento dos portos para o cuidado à saúde dos trabalhadores.

As ações direcionadas a assistência necessitava de um sistema previdenciário desenvolvido, sendo constituídas, inicialmente, as Caixas de Aposentadorias e Pensões (CAPs) que posteriormente seriam substituídas pelos Institutos de Aposentadorias e Pesões (IAPs), que era um sistema previdenciário organizado por categorias profissionais (RONCALLI, 2003). Desta forma se inicia a assistência odontológica privada, através de contribuições financeiras as CAPs e em seguida aos IAPs. Algumas CAPS e posteriormente IAPs desenvolveram algum tipo de assistência odontológica aos seus beneficiários, com o surgimento dos consultórios que ofereciam pelo menos atendimento emergencial (LOURENÇO, 2003), sem dar ênfase aos aspectos de promoção e prevenção em saúde bucal para a população.

É possível que esta mudança de eixo de atenção tenha proporcionado novos conhecimentos clínicos e epidemiológicos e se estabelecido como princípio da política de saúde brasileira, pois pela necessidade de manter saudáveis os trabalhadores, o Estado passa a atuar de forma mais duradoura e não $\begin{array}{llll}\text { apenas no } & \text { controle }\end{array}$ epidemias(LOURENÇO, 2003).A Fundação 
Serviços Especiais de Saúde Pública (FSESP) nos anos 1950, que possuía atendimento a escolares, desenvolvendo ações odontológicas através de várias instituições, contribuiu, desta forma, para a busca pela estruturação da assistência odontológica no país (RONCALLIet al., 1999).

Neste período, a FSESP implementa o Sistema Incremental (SI) de Atenção ao Escolar e em 1964, quando ocorre a III Conferência Nacional de Saúde, inclui a prevenção da cárie dentária pelo uso do flúor no Plano Nacional de Saúde Pública (ELY; CARVALHO; SANTOS, 2009). Com o SI, inicia-se a prevenção a doença cárie.

Mesmo com o desenvolvimento e a realização de atividades por outros modelos gerenciais, o que se estabeleceu como modelo hegemônico foi o SI em escolares, devido aos grandes avanços em relação à assistência odontológica na época. Nos anos 70, o SI desenvolvia assistência odontológica somente para escolares de 7 a 14 anos (ELY; CARVALHO;

SANTOS, 2009), proporcionando uma atenção odontológica somente a uma parte da população, excluindo um grande número de pessoas que também necessitavam de assistência, levando este modelo a ser considerado ultrapassado já no inicio da década de 1970 (RONCALLIet al., 1999). O SI não conseguiu reduzir a incidência de cárie dentária, sendo alcançado um aumento no número de dentes restaurados sem nenhum impacto sobre os níveis de saúde bucal, por ser um modelo preventivo que possuía um curto alcance e era realizado semmetodologias

efetivas(LOURENÇO,2003).

Sua ação levou a um aumento do número de dentes restaurados, através de sua filosofia curativo-reparador, não melhorando, porém, os níveis de saúde bucal da população (RONCALLIet al.,1999). Esta filosofia contribuiu para aumentar o nível de edentulismo da população, pois além deste procedimento, prevalecia a prática de extrações dentárias, caracterizando uma atividade mutiladora.

Em 1974, através da promulgação da Lei Federal 6050, se tornou obrigatória a fluoretação das águas de abastecimento público em nível nacional (BRASIL, 1974; ELY; CARVALHO; SANTOS, 2009), sendo uma medida de grande importância para a redução do nível de lesões de cárie na população. Durante as décadas de 1970 e 1980 buscou-se implementar a fluoretação das águas de abastecimento, mas esta luta ocorre até hoje, pois atualmente a carência de recursos, iniciativa política e as dificuldades impostas pelas Companhias de Abastecimento dificultam a sua implantação a serviço da população (LOURENÇO, 2003).Outras ações como o Programa de Interiorização das Ações de Saúde e Saneamento do Nordeste (PIASS), criado em 1976, ofertavam ações de saúde bucal somente para a população adulta 
(atenção mutiladora e em urgências) (ELY; CARVALHO; SANTOS, 2009), sem mudança do enfoque centrado em determinadas faixas etárias, excluindo parte da população. No início dos anos de 1980, devido à assistência odontológica ter expandido no setor público, foi criado o Plano de Reorientação da Assistência Odontológica (PRAOD) pelo Conselho Consultivo da Administração de Saúde Previdenciária (CONASP) (LOURENÇO, 2003).

Neste período, o Programa de Ações Integradas de Saúde (PAIS) no CONASP enviou para vários municípios verbas para aquisição de equipamentos odontológicos nas escolas de primeiro grau e o pagamento dos salários dos profissionais (ELY; CARVALHO; SANTOS, 2009). Ainda nesta década o Instituto Nacional de Assistência Médica e Previdência Social (INAMPS) aumentou paulatinamente os repasses financeiros para as instituições públicas, com ênfase na atenção odontológica escolar, visando desta forma um equilíbrio de gastos entre prestadores públicos e privados (LOURENÇO, 2003).A realização da I Conferência de Saúde Bucal, ocorrida no mesmo período da VIII Conferência Nacional de Saúde (1986), abordou assuntos relevantes das condições da atenção odontológica da população, considerando a saúde bucal como parte integrante e inseparável da saúde geral do indivíduo (ELY; CARVALHO; SANTOS,
2009), associação não estabelecida anteriormente.

\section{POLÍTICAS DE SAÚDE BUCAL NO CONTEXTO DO SISTEMA ÚNICO DE SAÚDE}

Com a criação do SUS, este veio proporcionar uma base filosófica e programática que direciona para a mudança do modelo de atenção (medico assistencial privatista, de paradigma mecanicista), visando $\mathrm{o}$ acesso e atendimento para toda a população brasileira (RONCALLIet al., 1999).

A Política Nacional de Saúde Bucal foi aprovada através da Portaria GM no 613/89, em junho de 1989 (BRASIL, 1989), logo após a promulgação da Constituição da Republica Federativa do Brasil. Inicialmente priorizava as crianças em idade de escolarização primaria (de 6 a 12 anos), do ponto de vista de grupos populacionais (BRASIL, 2009). Em sequência, seriam atendidas as faixas etárias dos adolescentes (13 a 19 anos), crianças de 2 a 5 anos e adultos, as ações preventivas e de educação em saúde bucal e aos serviços recuperadores básicos. Como quarta e quinta prioridades, estavam às especialidades básicas (endodontia, cirurgia, ortodontia e prótese) e os serviços especializados de maior complexidade(BRASIL, 2009).

Em 1993, na II Conferência Nacional de Saúde Bucal (CNSB), discutiu-se uma 
nova política de saúde bucal com inserção no SUS, garantindo o acesso e a equidade da assistência odontológica

(ELY;

CARVALHO; SANTOS， 2009).A saúde bucal coletiva no Brasil acompanhou $\mathrm{O}$ processo de construção e implantação do SUS na elaboração de políticas públicas, buscando a transformação da prática de assistência odontológica, caracterizada por ser ineficaz, ineficiente, de baixa cobertura, de caráter capitalista, com baixa resolubilidade e distribuída geográfica e socialmente desiguais(JUNQUEIRA;

FRIAS; ZILBOVICIUS, 2004).

A incorporação da Equipe de Saúde Bucal (ESB) na Estratégia de Saúde da Família (ESF), em 2000, possibilitou uma maior atuação da odontologia na atenção primária em saúde, pois além do atendimento clínico, atuam na comunidade realizando levantamentos epidemiológicos, ações preventivas e de educação em saúde, buscando a promoção de saúde bucal (ANTUNES; NARVAI, 2010).

Com os resultados alarmantes das condições de saúde bucal da população, diagnosticados pelo levantamento de Saúde Bucal do Brasil (SB Brasil 2003), em 2004 foi lançada a Política Nacional de Saúde Bucal (Brasil Sorridente), a qual reforça a inserção das ESB na ESF, criando os Centros de Especialidades Odontológicas (CEO) e expandindo a cobertura da fluoretação da água de abastecimento público (BRASIL,
2004 ${ }^{\mathrm{a}}$; ELY; CARVALHO; SANTOS, 2009)A III CNSB com o tema central "Saúde Bucal: acesso e qualidade, superando a exclusão social", se realizava neste período dentro de um contexto de grande expectativa para a população, pois reivindicações históricas poderiam se tornar realidade, principalmente as relacionadas a saúde bucal (BRASIL, 2005).

A Portaria GM $\mathrm{n}^{0}$ 1.570/2004estabeleceu várias condições para a implantação e credenciamento de CEO e Laboratórios Regionais de Próteses Dentarias (LRPD), em que as atividades como diagnostico bucal, periodontia, cirurgia oral menor dos tecidos moles e duros, endodontia e atendimento a portadores de necessidades especiais, devem ser realizadas pelo CEO(BRASIL, 2004b).

A partir da implantação dos CEO e LRPD, os usuários começam a ter um maior acesso à reabilitação de suas perdas dentárias, principalmente desenvolvidas pela prática mutiladora, ausência de ações de promoção e prevenção em saúde bucal. Estas condições resultaram em altos níveis de perdas dentárias na população.Embora o edentulismo não seja considerado um problema de saúde pública, este deve ser considerado como tal, pois há a necessidade de modificação da ideia de que a perda dentária seja uma consequência natural do envelhecimento(JUNQUEIRA; $\quad$ FRIAS; ZILBOVICIUS, 2004). 
A atenção secundária em odontologia, desempenhada pelos os CEOs e pelos LRPD, tem realizado reabilitações através de próteses parciais e totais. Mas, é necessário fiscalizar o modo de produção destas, pois se pode encontrar indivíduos reabilitados que possuem limitações em suas atividades, como sorrir, comer e falar, em diferentes graus de impactos (MACHADOet al., 2013). Um estudo relatou (COSTAet al., 2013) um alto índice de próteses totais convencionais superiores e inferiores insatisfatórias tecnicamente, atingindo $47,3 \%$ e $90,5 \%$, respectivamente. Assim, estes aparelhos reabilitadores podem gerar Disfunções Temporomandibulares (DTMs) e lesões estomatológicas em curto ou em longo prazo.

A reabilitação dos dentes perdidos pelos usuários é de suma importância, pois além de restabelecer a função, estética, fonética, busca o restabelecimento do equilíbrio da oclusão. Este é de suma importância, pois os fatores oclusais estão envolvidos na etiologia das DTMs(BARBOSAet al., 2003; SEABRA; BARBOSA; LIMA, 2012). A disfunção temporomandibular (DTM) é considerada um conjunto de alterações articulares e musculares que acometem a região orofacial (ALMEIDAet al., 2008). Os sinais e sintomas comuns da DTM são a redução dos movimentos mandibulares, diminuição da função da Articulação temporomandibular (ATM), presença de dor ou sensibilidade muscular à palpação, dor durante o movimento mandibular, dores faciais, cefaléia e ruídos articulares, sendo estes últimos os mais prevalentes (MCNEILLet al., 1980; ALMEIDA et al., 2008;SEABRA; BARBOSA; LIMA, 2012).

Devido a sua etiologia multifatorial, podem estar envolvidos em seu desenvolvimento os fatoresoclusais, traumáticos, alterações articulares e musculares, hábitos parafuncionais (bruxismo) e estresse emocional e físico, os quais irão alterar o estado de normalidade do sistema estomatognático, podendo gerar a disfunção ～(MARTINS， 2008;SEABRA; BARBOSA; LIMA, 2012). A prevalência de DTM tem sido extensivamente estudada em várias faixas etárias e localidades, mas os resultados dos estudos em população idosa são ainda inconsistentes (SCHMITTER; RAMMELSBERG; HASSEL， 2005). Os primeiros estudos relacionados à DTM foram com indivíduos dentados, sendo que por um longo período os indivíduos desdentados não foram pesquisados, e ainda, atualmente, as publicações com relevância referentes à prevalência de DTM nestes indivíduos, portadores ou não de próteses totais, são escassas e controversas, não sendo possível obter uma conclusão fidedigna (BONTEMPO; ZAVANELLI, 2011). Portanto, há a necessidade de estudos para avaliar a condição dentária de pacientes portadores de DTM, como também em 
pacientes não portadores de DTM. A relação entre condição dentária e DTM é pouco relatada nas bases de dados eletrônicos e

\section{CONSIDERAÇÕES FINAIS}

A prática odontológica que até os dias atuais está presente, caracterizada ainda como mutiladora, com enfoque biológico, tecnicista e pouco resolutiva, tem contribuído pouco para a melhoria das condições de saúde bucal das populações, desta forma sem garantir uma qualidade de saúde bucal da população. Assim, é necessário um processo de reorganização das atividades de Saúde Bucal, para cada vez mais atuar de forma resolutiva sobre os problemas de saúde bucal e geral da população. A inclusão das ESB na ESF proporcionou um processo de reorientação da atenção primária em saúde, visando uma maior relação entre as políticas de saúde e os programas e/ou estratégias de saúde bucal.

Esta inserção é um grande desafio a ser vencido, pois sua inclusão nas políticas de saúde pelos representantes do governo, gestores e profissionais de saúde e usuários, é

\section{REFERÊNCIAS}

ALMEIDA, L. H. M.et al. Disfunção temporomandibular em idosos.Revista da Faculdade de Odontologia - UPF, Passo Fundo , v.13, n.1, p.3538, 2008. emoutros meios de publicação literária, assim há uma carência de estudos neste assunto.

de suma importância para a população, pois se trata de um pré-requisito para se ter uma saúde geral.

Além de implantar serviços para confecções de próteses, na média complexidade em saúde bucal, há a necessidade de fiscalizar o modo de produção destas, pois no seu desenvolvimento pode ocorrer a não realização de etapas necessárias para uma satisfatória reabilitação, gerando um impacto negativo significativo na qualidade de vida dos indivíduos.

Um princípio de fundamental importância para vencer este desafio é a Participação popular, pois através do diagnóstico da situação de saúde bucal, a sociedade pode e deve contribuir para a construção e implementação de políticas de saúde que proporcione melhores níveis de saúde para a população, para que o país com mais profissionais formados em odontologia do mundo deixe de ser conhecido com o país dos desdentados.

ANTUNES, J. L. F.; NARVAI, P. C.. Políticas de saúde bucal no Brasil e seu impacto sobre as desigualdades em saúde. RevistadeSaúde Públicada USP: RSP, São Paulo, v.44, n.2, p. 360-365, 2010.

BARBOSA, G. A. S.et al. Distúrbios oclusais: associação com a etiologia ou uma conseqüência das disfunções temporomandibulares? Jornal Brasileiro de 
Oclusão, ATM e Dor Orofacial, Curitiba, v.3, n.10, p.158-163, 2003.

BONTEMPO, K. V.; ZAVANELLI, R. A. Desordem temporomandibular: prevalência e necessidade de tratamento em pacientes portadores de próteses totais duplas.Revista Gaúcha de Odontologia, Porto Alegre, v.59, n.1, p.87-94, 2011.

BRASIL. Ministério da Saúde. Saúde Brasil 2008: 20 anos de Sistema Único de Saúde (SUS) no Brasil. Brasília, 2009. 154-174p.

BRASIL. Ministério da Saúde. 3. a Conferência Nacional de Saúde Bucal: acesso e qualidade superando exclusão social. Brasília, 2005. 148p.

BRASIL. Ministério da Saúde. Diretrizes da politica nacional de saúde bucal. Brasília, 2004a. 16p.

BRASIL. Ministério da Saúde. Portaria GM/MS n. 1570.Brasília, 2004b. 2p.

BRASIL. Ministério da Saúde. Politica nacional de saúde bucal. Brasília, 1989.

BRASIL. Ministério da Saúde. Lei ${ }^{\circ}$ 6.050, de 24 de maio de 1974. Brasília, 1974. 1p.

BUSS, P. M.; PELLEGRINI FILHO, A.A Saúde e seus Determinantes Sociais. PHYSIS: Revista de Saúde Coletiva, Rio de Janeiro, v.17, n.1, p.77-93. 2007.

CAMPOS, G. W. S. Efeitos paradoxais da descentralização no sistema único de saúde do brasil. In: FLEURY, S.; CAMPOS, G. W. S. (Org.). Democracia, descentralização e desenvolvimento: Brasil \& Espanha. São Paulo: EditoraFundação Getúlio Vargas, 2006, 417-442.
CARVALHO, C. L.Dentistas práticos no Brasil: história de exclusão e resistência na profissionalização da odontologia brasileira. Tese (Doutorado) - Escola Nacional de Saúde Pública da Fundação Oswaldo Cruz, Rio de Janeiro. 2003.

COSTA, A. P. S.et al. Qualidade técnica e satisfação relacionadas às próteses totais. RevistaCiência \& Saúde Coletiva, Rio de Janeiro, v.18, n.2, p.453-460. 2013.

ELY, H. C.; CAVALHO, D. Q.; SANTOS, M.POLÍTICAS DE SAÚDE BUCAL. Texto preliminar, de circulação interna, escrito para subsidiar a elaboração dos Cadernos de Atenção Básica do Departamento de Atenção Básica do Ministério da Saúde.Saúde bucal. Mimeo. Brasília/Porto Alegre, v.17,2006. Atualizado em 2009. Disponível em:https://www.nescon.medicina.ufmg.br/biblioteca/i magem/2200.pdf. Acesso em: 20 dez. 2013.

JUNQUEIRA, S. R.; FRIAS, A. C.; ZILBOVICIUS, C.Saúde bucal coletiva: quadros social, epidemiológico e político.In: RODE, S. M.; NUNES, S. G. (Org.).Atualização clínica em odontologia. São Paulo: Editora Artes Médicas, 2004, p. 591-604.

LOURENÇO, E. C.O processo histórico de construção das políticas públicas de saúde no brasil.Monografia (Especialização) - Faculdade de Odontologia, Universidade Estadual de Campinas, Piracicaba. 2003.

MACHADO, F. C. A.Dificuldades diárias associadas às próteses totais. RevistaCiência \& Saúde Coletiva, Rio de Janeiro, v.18, n.10, p.3091-3100, 2013.

MARTINS, R. J.et al. Relação entre classe socioeconômica e fatores demográficos na ocorrência da disfunção temporomandibular. Revista Ciência \& 
Saúde Coletiva, Rio de Janeiro, v.13, n.2, p.2089-2096, 2008.

MCNEILL, C. et al. Craniomandibular (TMJ) disorders: the state of the art. The Journal of Prosthetic Dentistry, Augusta, v.44, n.4, p.434-437, 1980.

MOTT, M. L. et al. 'Moças e senhoras dentistas': formação, titulação e mercado de trabalho nas primeiras décadas da República. História, Ciências, Saúde - Manguinhos, Rio de Janeiro, v.15, Supl., p.97-116, 2008.

PEREIRA, W.Uma história da odontologia no brasil. História e Perspectivas, Uberlândia, v.47, p.147-173, 2012.

RONCALLI, A. G. O desenvolvimento das políticas públicas de saúde no Brasil e a construção do Sistema Único de Saúde. In: PEREIRA, A. C. (Org.).Odontologia em Saúde Coletiva: planejando ações e promovendo saúde. Porto Alegre: Editora Artmed, 2003, p.28-49.
RONCALLI, A. G. et al. Modelos assistenciais em saúde bucal no Brasil: tendências e perspectivas. RevistaAçãoColetiva, Brasília, v.2, n.1, p.9-14,1999.

SCHMITTER, M.; RAMMELSBERG, P.; HASSEL, A.The prevalence of signs and symptoms of temporomandibular disorders in very old subjects.Journalof Oral Rehabilitation, Malden, v.32, n.7, p.467-473, 2005.

SILVA, R. H. A.; SALES-PERES, A.Odontologia: Um breve histórico. Odontologia Clínico-Científica, Recife, v.6, n.1, p.7-11, 2007.

SEABRA, E. J. G.;BARBOSA, G. A. S.; LIMA, I. P. C.Oclusão e DTM:Conhecimentos aplicados à Clínica Odontológica. $1^{\mathrm{a}}$ ed. Natal: Editora Edições UERN. 2012.

VARGAS, J. D. História das Políticas Públicas de Saúde no Brasil: revisão da literatura. 2008. 32p. Trabalho de Conclusão de Curso (especialização) Escola de Saúde do Exército, Rio de Janeiro, 2008.

WARMLING, C. M.; CAPONI, S.; BOTAZZO, C. Práticas sociais de regulação da identidade do cirurgião-dentista. Revista Ciência \& Saúde Coletiva, Rio de Janeiro, v.11, n.1, p.115-122. 2006. 